\title{
Changes in the mandibular angle during adulthood in South Africans
}

\author{
A.C. Oettlé, ${ }^{1 *}$ R. Ehlers, ${ }^{2}$ and M. Steyn, ${ }^{1,3}$ \\ ${ }^{1 .}$ Department of Anatomy, School of Medicine, Faculty of Health Sciences, University of \\ Pretoria, Pretoria, South Africa \\ 2. Department of Statistics, Faculty of Natural and Agricultural Sciences, University of \\ Pretoria, Pretoria, South Africa \\ 3. School of Anatomical Sciences, Faculty of Health Sciences, University of the \\ Witwatersrand, Johannesburg, South Africa
}

Running headline: Changes in the mandibular angle in adults

*Corresponding author: Dr AC Oettlé

Telephone: +27 $123192430 /+27838702379$

Fax nr: +27 123192240

Email address: anna.oettle@up.ac.za

Department of Anatomy

School of Medicine

Faculty of Health Sciences

University of Pretoria

Private Bag x 323

ARCADIA 0007

Pretoria

Republic of South Africa

Dr AC Oettlé received funding for relief staff as part of the Vice-chancellor's academic development grant programme towards the PhD protocol number: 120/2010. The research of Prof Steyn is supported by the NRF (National Research Foundation: South Africa). 


\begin{abstract}
Objectives: The purpose of this study was to measure the mandibular angle as a reflection of the biomechanical forces acting on the mandible and relate it to aging and loss of teeth.

Methods: A total of 717 mandibles of adult individuals were selected from the Pretoria Bone Collection. These mandibles were allocated to dentition subgroups according to their pattern of tooth loss. The angle of the mandible was measured with a mandibulometer. The variation in the mandibular angle was statistically analyzed by age regressions and comparisons between adjusted means of dentition subgroups.

Results: Aging per se was not associated with a greater mandibular angle. With limited tooth loss, aging was associated with a smaller mandibular angle in females. Generally the mandibular angle increased with tooth loss and was accentuated when an even occlusion pattern was lost rather than with more extensive tooth loss. A more pronounced increase in mandibular angle with tooth loss was noted in females and individuals of European ancestry.

Conclusions: Tooth loss and not aging is associated with an increase in the mandibular angle. Masculinity and being from African ancestry provided some protection against an increase in the mandibular angle with tooth loss. The accentuated increase in the mandibular angle when an even occlusion pattern was disturbed supports the notion that an uneven loss of teeth was indeed associated with inefficient mastication. The unexpected decrease in angle noted in females with aging but limited tooth loss may be indicative of constructive changes beyond puberty.
\end{abstract}

Key Words: occlusions; uneven tooth loss; mandibulometer; aging; biomechanical forces

\title{
INTRODUCTION
}

The direct measurement of the mandibular angle, also called the gonial angle, is a well-known parameter used in the forensic setting and for planning dentistry procedures (De Villiers, 1968; Moore-Jansen et al., 1994; Xie and Ainamo, 2004; Parr, 2005; Franklin et al., 2008a; Osato et al., 2012). It is recorded with an established, simple technique. The size of the mandibular angle is often thought of as representing the influence of biomechanical factors on the morphology of the mandible (Da Costa De Sousa et al., 2013). The main 
elevator muscles of the mandible - the masseters and the medial pterygoids - act together as a sling around the angle of the jaw to lift the mandible and to close the space between the upper and lower jaws (Potgieter et al., 1983). A greater muscular force would result in a more acute angulation of the mandibular angle, while the angulation in response to a lesser force and thus reduced functionality would be more obtuse (Keen, 1945; Kasai et al., 1996; Ohm and Silness, 1999).

The mandibular angle is considered to be formed by the ramus line (the tangent to the posterior border of the mandible) and the mandibular line (the relatively straight portion of the inferior border of the mandible that ends posterior to the gnathion - the most anterior midline point on the mandible) (Tobias, 1974; Ohm and Silness, 1999).

The size of the mandibular angle has been considered to become obtuse in old age and therefore more similar to the infantile angle of $135^{\circ}$ to $150^{\circ}$ (Jensen and Palling, 1954). This is not unexpected as senescence has a general degenerative effect on the skeleton and specifically on the morphology of the mandible (Doual et al., 1997). However, these changes in the mandible are not clearly understood as they are not only associated with senescence but may also be accounted for by tooth loss (Keen, 1945; Ohm and Silness, 1999; Xie and Ainamo, 2004).

As tooth loss may influence the efficacy of mastication and therefore the biomechanical factors acting on the mandible, changes in the mandibular angle with tooth loss would not be unexpected. Potgieter et al. (1983), considered the mandibular angle as an anatomical feature that would be unaffected by the loss of teeth (in the short term) but with time would be subject to modification by the effect of muscular force. In edentulous people, changes in the mandibular bone after tooth extraction may present as a widening of the mandibular angle (Kohakura et al., 1997). However, not all authors agree with the idea that the mandibular angle is influenced by tooth loss (Israel, 1973; Enlow et al., 1976). Xie and Ainamo (2004), for instance, found no association between angle size and duration of edentulism. In a study by Ceylan et al. (1998) no significant difference was found between the dentulous group and those with unilateral and posterior tooth loss, but Oettlé et al. (2009) found that a unilateral loss of molars may have an even greater effect than bilateral loss. When all molar teeth were present or the loss of teeth was bilateral, a more efficient chewing mechanism was created with a more optimal action of the masticatory muscles, inducing the more acute angle of the ramus of the mandible (Oettlé et al., 2009). Even, simultaneous and bilateral tooth contacts in the intercuspal position are assumed to provide a balanced distribution of occlusal force (Pileicikiene and Surna, 2004; Ceylan et al., 1998). 
The purpose of this study was to assess the size of the mandibular angle as a reflection of the biomechanical forces acting on the mandible and relate it to aging and the loss of teeth. Statistical assessments were designed to distinguish between the effect of aging and tooth loss in the two sexes and ancestral groups studied. Groups showing progressive tooth loss were defined so as to enable a possible separation of three biomechanical scenarios which could influence the mandibular angle. This differentiation could have implications for the planning of restorative dentistry procedures, e.g. to retain teeth at pivotal locations and to design dentures (Potgieter et al., 1983; Moipolai et al., 2003; Xie and Ainamo, 2004).

The notion that unbalanced loss of molars has a negative effect on the function of mastication and associated acuteness of the mandibular angle, rendering it more obtuse (Oettlé et al., 2009), was implemented along with other guidelines from the literature to construct criteria to define dentition groups which might better represent biomechanical forces on the mandible. Apart from the number and position of the remaining teeth, occlusions of the mandibular teeth with the corresponding maxillary teeth were also considered to define groups with progressive tooth loss (Kasai et al., 1994; Kasai et al., 1996; Kohakura et al., 1997; Ceylan et al., 1998; Pileicikiene and Surna, 2004; Yanıkoğlu and Yılmaz, 2008; Oettlé et al., 2009).

As masticatory function does not only influence the mandibular angle but also other aspects of the morphology of the mandible, the size of the mandibular angle might also be useful to predict, for example, cortical thickness that is very important in implantology (Xie and Ainamo, 2004; Osato et al., 2012; Da Costa De Sousa et al., 2013). Variations between ancestral groups and sexes in the mandibular angle with aging and tooth loss may also prove to be a useful adjunct in the identification of unknown remains if they are sufficiently distinct to provide demographic information.

The aim of this study therefore was to relate the size of the mandibular angle to aging and the loss of teeth. The null hypothesis was that the mandibular angle will increase with aging and tooth loss. The null hypothesis would be rejected if any group did not show an increase in the size of the mandibular angle with aging or tooth loss.

\section{MATERIALS}

A total of 717 mandibles from individuals between the ages of 18 and 98 years were selected from the Pretoria Bone Collection (L’Abbé et al., 2005). These are all modern, known individuals deriving from cadaver dissection. Adult mandibles without obvious 
deformity or surgery, but with varying degrees of dental loss and documented age, sex and ancestral group were included. Two sexes and ancestral groups were considered: South Africans of African ancestry (SAA) and South Africans of European ancestry (SAE).

The age distribution amongst sex-ancestral-dentition groups for the mandibular angle is set out in Table 1 .

TABLE 1. Age distribution in years amongst sex-ancestral-dentition groups for the mandibular angle $(n=717)$

\begin{tabular}{|c|c|c|c|c|}
\hline \multirow{3}{*}{ Dentition groups $\downarrow$} & \multicolumn{4}{|c|}{ Ancestry and sex } \\
\hline & \multicolumn{2}{|c|}{ SAA } & \multicolumn{2}{|c|}{ SAE } \\
\hline & Female & Male & Female & Male \\
\hline \multirow{4}{*}{ Group 0} & 9 & 90 & 69 & 90 \\
\hline & {$[32-75]$} & [31 - 98] & [48 - 97] & {$[40-91]$} \\
\hline & 57.44 & 68.11 & 74.64 & 72.84 \\
\hline & $(14.32)$ & $(11.32)$ & $(10.33)$ & $(11.10)$ \\
\hline \multirow{4}{*}{ Group 1} & 15 & 138 & 11 & 22 \\
\hline & {$[26-80]$} & {$[24-80]$} & {$[56-88]$} & {$[38-89]$} \\
\hline & 49.27 & 58.70 & 70.91 & 66.64 \\
\hline & $(17.56)$ & $(11.68)$ & $(11.70)$ & $(13.44)$ \\
\hline \multirow{4}{*}{ Group 2} & 35 & 204 & 16 & 18 \\
\hline & {$[21-70]$} & {$[18-87]$} & {$[21-87]$} & {$[36-79]$} \\
\hline & 43.57 & 51.06 & 66.69 & 63.22 \\
\hline & $(12.93)$ & $(15.21)$ & $(16.48)$ & $(10.86)$ \\
\hline
\end{tabular}

$\mathrm{n}$ the first value in every set

minimum and maximum values in square brackets [] mean given in bold

standard deviation in parenthesis ()

\section{METHODS}

The protocol and procedures employed were reviewed and approved by the Ethics Committee of the Faculty of Health Sciences of the University of Pretoria.

In this cross-sectional study the number and location of the mandibular teeth as well as their occlusion with upper teeth were recorded. Established determinants for the efficiency of mastication with regard to tooth loss pattern include whether or not posterior and anterior teeth are in occlusion and whether they are evenly distributed between the left and the right (Kasai et al., 1994; Kasai et al., 1996; Kohakura et al., 1997; Ceylan et al., 1998; Pileicikiene and Surna, 2004; Yanıkoğlu and Y1lmaz, 2008; Oettlé et al., 2009). The before mentioned determinants for efficacy of mastication with regard to the position and occlusion of teeth were taken into consideration in the design of the study. Criteria were constructed to define three dentition subgroups: group 0: a group with no mandibular teeth in occlusion with corresponding maxillary teeth; group 1: a group with fewer than two posterior teeth (molar and premolar teeth) and/or no front teeth (incisors and canine) in occlusion on each side; and 
group 2: a group with two or more posterior teeth (molar and premolar teeth) and one or more front teeth (incisors and canine) in occlusion on each side.

The angle of the mandible was measured with a mandibulometer (GPM Gneupel, Switzerland) according to the standard method as described by Jensen and Palling (1954), De Villiers (1968), and Srisopark (1975) (Fig. 1). Following De Villiers (1968), all measurements were taken with the mandibles placed on the horizontal surface with the teeth facing up. The mandible was adjusted so that the horizontal part of the board represented the standard horizontal plane, whereas the rameal (movable) wing of the board was in contact with the posterior surface of the left ramus at the condyle and inferiorly above the angle and with the right ramus at one or both of these regions. The mandible was therefore in such a position that three or more points made contact with the rameal plane of the mandibulometer when vertical pressure was applied to the left second molar tooth or its cavity. The left angle between the standard horizontal and standard rameal planes (the rameal wing of the board) was read on the protractor.

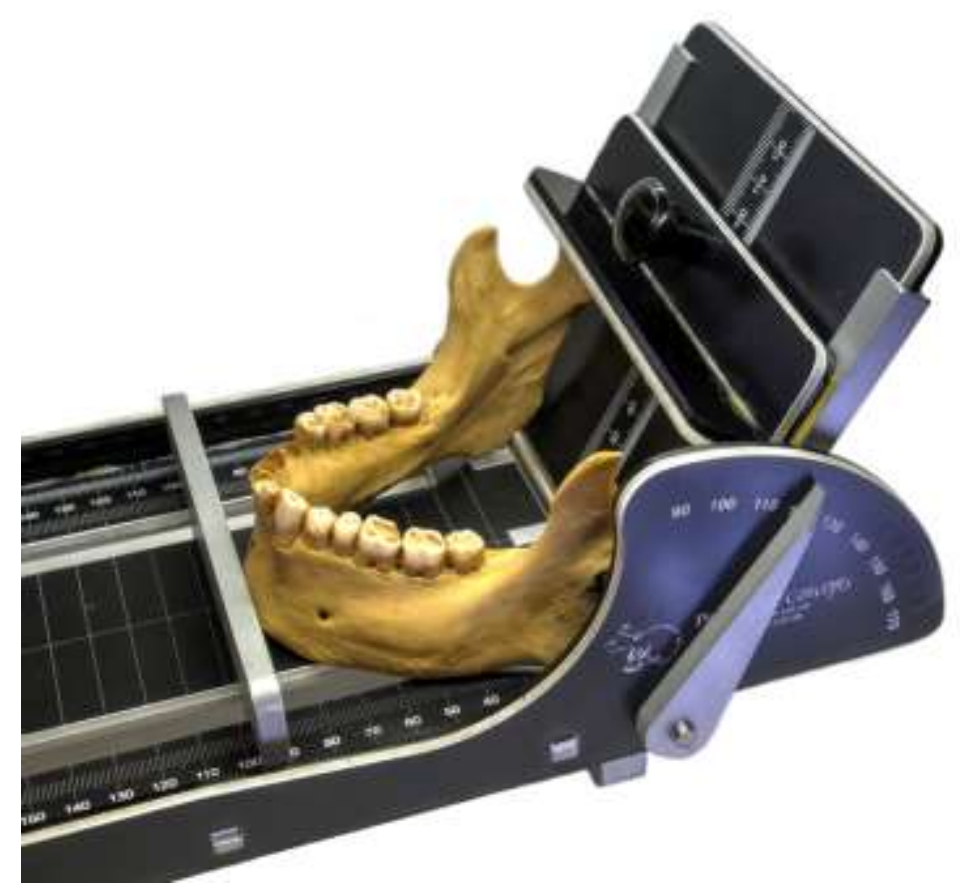

Fig. 1. Measuring the mandibular angle with a mandibulometer

The relationship between mandibular angle and age was investigated for each sexancestral-dentition group by calculating Pearson correlation coefficients. An ANCOVA was performed for each sex-ancestral group to test for differences in the means of the mandibular angle amongst various dentition groups. Age was entered as a covariate and least square 
means were calculated. In groups where the mean differed, pairwise comparisons of the least square means were calculated. In these groups, pairwise comparisons of the means were done with a Bonferroni correlation for multiple comparisons.

Interrater reliability of the measurements taken by a second observer familiar with the technique was tested on a random sample of 30 specimens and measured by the Maximum Likelihood Estimate of the Intraclass Correlation Coefficient. Similarly, Intrarater reliability of the measurements of the main investigator was tested when the measurements were repeated at a later stage.

\section{Results}

Overall, within the four sex-ancestral groups, weak to negligible correlations between mandibular angle size and age existed. To isolate the changes with aging in the four sexancestral groups to a specific dentition group, the 12 sex-ancestral-dentition groups (shown in Table 1) were further analyzed by linear regressions of mandibular angle vs. age. The decrease in mandibular angle size with age in females of both SAA and SAE was the most pronounced in dentition group 2 in females and statistically significant in female SAA (female SAA: $r=-0.40 ; p=0.02$ ) (Fig.2). Only weak to very weak associations existed between mandibular angle and age in males.

Basic descriptive statistics were performed by univariate analysis, producing crude mean values of the mandibular angle for the 12 sex-ancestral-dentition groups (Table 2). Increases in the mandibular angle with progressive loss of teeth were most noticeable in mandibles of SAE (South Africans of European ancestry).

In Table 3 the least square means of the mandibular angle across ancestral group, sex and dentition groups are given corrected for age, a continuous variable. Although adjustments for age were made, sex-ancestral group differences in the period from tooth loss to death could not be regulated.

An increase in the mandibular angle was noted with progressive tooth loss. In most groups the difference in mandibular angle was accentuated between dentition group 2 and 1 , indicating that the mandibular angle changes occurred even with initial tooth loss. It is interesting to note that the size of the mandibular angle in dentition group 1 was also often the greatest for the particular sex-ancestral group. Greater levels of variance (less precise estimates) were evident from the larger confidence intervals in females as compared to males and SAE compared to SAA (Table 3). Confidence intervals of dentition groups overlapped each other within each sex-ancestral group and were often the greatest in dentition group 1. 

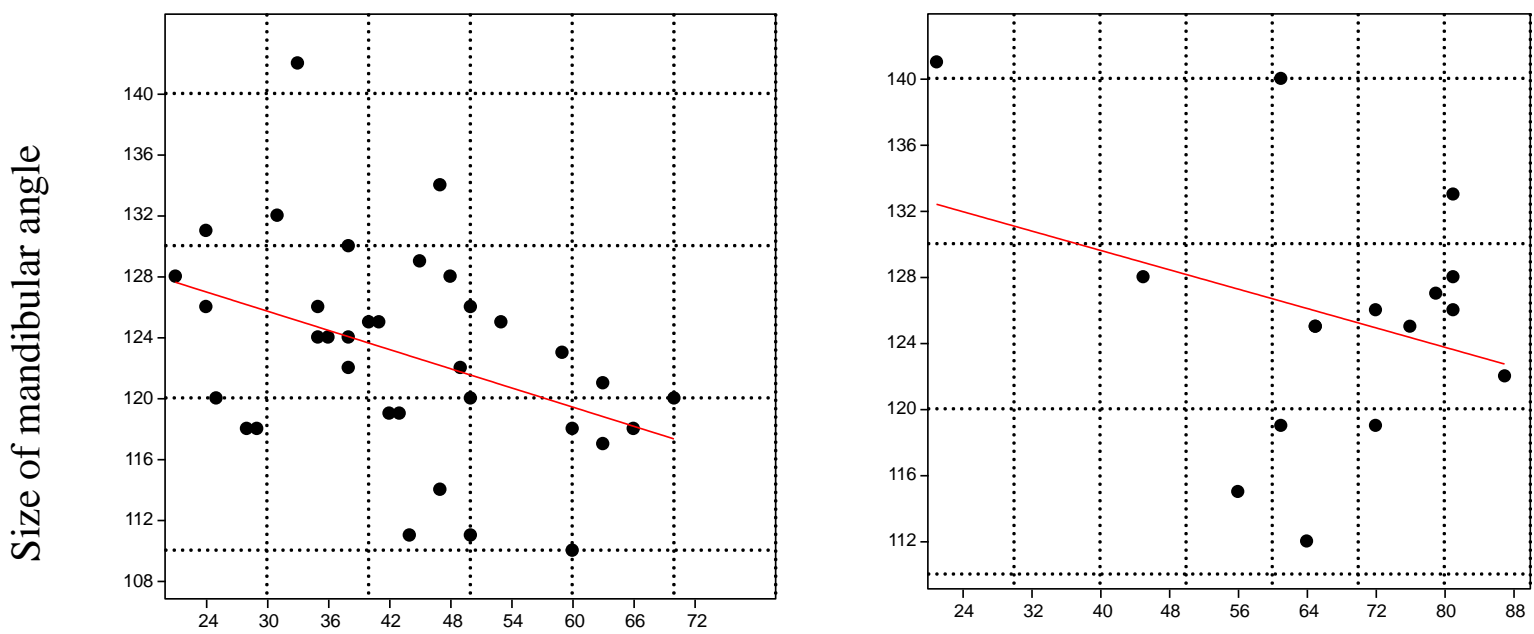

Age in years

Fig. 2. Linear regression of age in years vs. size of mandibular angle in females

TABLE 2. Univariate analysis of the mandibular angle for sex-ancestral-dentition groups

\begin{tabular}{lllll}
\hline \multirow{2}{*}{$\begin{array}{l}\text { Dentition } \\
\text { groups } \downarrow\end{array}$} & Ancestry and sex & & \\
\cline { 2 - 5 } & SAA & & SAE & \\
& Female & Male & Female & Male \\
\hline \multirow{2}{*}{ Group 0 } & 9 & 90 & 69 & 90 \\
& $\mathbf{1 2 3 . 4 4}$ & $\mathbf{1 2 1 . 0 6}$ & $\mathbf{1 2 7 . 5 5}$ & $\mathbf{1 2 5 . 5 0}$ \\
& $(7.43)$ & $(8.36)$ & $(7.22)$ & $(6.95)$ \\
\hline \multirow{2}{*}{ Group 1 } & 15 & 138 & 11 & 22 \\
& $\mathbf{1 2 4 . 4 7}$ & $\mathbf{1 2 1 . 6 2}$ & $\mathbf{1 2 8 . 2 7}$ & $\mathbf{1 2 3 . 7 3}$ \\
& $(4.34)$ & $(7.97)$ & $(6.03)$ & $(8.89)$ \\
\hline \multirow{2}{*}{ Group 2 } & 35 & 204 & 16 & 18 \\
& $\mathbf{1 2 2 . 8 6}$ & $\mathbf{1 2 0 . 3 0}$ & $\mathbf{1 2 5 . 6 9}$ & $\mathbf{1 2 3 . 1 7}$ \\
& $(6.74)$ & $(6.93)$ & $(7.80)$ & $(6.38)$ \\
\hline
\end{tabular}

$\mathrm{n}$ the first value in every set

mean given in bold

standard deviation in parenthesis ()

The least square means between dentition groups, however, only differed significantly on the $10 \%$ level of significance $(\mathrm{p}=0.0514)$ when comparing group 0 and group 2 of female SAA. 
TABLE 3. Least square means of the mandibular angle adjusted for age and cell imbalances when dentition groups are compared within sex-ancestral groups

\begin{tabular}{|c|c|c|c|c|}
\hline \multirow{3}{*}{$\begin{array}{l}\text { Dentition groups } \\
\downarrow\end{array}$} & \multicolumn{4}{|l|}{ Ancestry and sex } \\
\hline & \multicolumn{2}{|l|}{$\overline{\mathrm{SAA}}$} & \multicolumn{2}{|l|}{ SAE } \\
\hline & Female & Male & Female & Male \\
\hline \multirow{2}{*}{ Group 0} & $124.85 *$ & 120.55 & 127.36 & 125.34 \\
\hline & $(122.86 ; 130.44)$ & $(118.99 ; 122.12)$ & $(125.67 ; 129.05)$ & $(123.91 ; 126.78)$ \\
\hline \multirow{2}{*}{ Group 1} & 124.76 & 121.59 & 128.17 & 123.96 \\
\hline & $(122.03 ; 127.39)$ & $(120.40 ; 122.78)$ & $(124.00 ; 132.34)$ & $(120.92 ; 127.00)$ \\
\hline \multirow{2}{*}{ Group 2} & $121.92^{*}$ & 120.29 & 125.35 & 123.60 \\
\hline & $(120.11 ; 123.72)$ & $(119.28 ; 121.30)$ & $(121.82 ; 128.89)$ & $(120.37 ; 126.84)$ \\
\hline $\begin{array}{l}\text { Dentition group } \\
\text { totals }\end{array}$ & 123. 32 & 120.76 & 127.12 & 124.88 \\
\hline \multicolumn{5}{|c|}{ adjusted means are indicated in bold. } \\
\hline \multicolumn{5}{|c|}{$95 \%$ confidence intervals are given within parentheses } \\
\hline
\end{tabular}

In summary, when sex-ancestral groups were evaluated individually, some increase in size of the mandibular angle was noted with loss of teeth in all. The greatest difference in angle size between groups with progressive tooth loss occurred in females of both ancestral groups followed by male SAE, with almost no difference in male SAA. A moderate negative correlation in the mandibular angle size existed with aging in females in dentition group 2. Apart from female SAA, age was not a significant co-variate when dentition groups were compared within sex-ancestral group. Tooth loss was accompanied by a significant increase in the mandibular angle in female SAA, counteracting the possible constructive effects of aging in the groups with limited tooth loss. The accentuated difference in mandibular angle between dentition groups 2 and 1, could be indicative that the dentition of group 1 was indeed inefficient.

Intraclass Correlation Coefficient was found to be 0.943 for intraobserver repeatability and 0.985 for interobserver repeatability, denoting excellent agreement. This shows that the measurement of the mandibular angle is straightforward and easily repeatable. 


\section{Discussion}

The null hypothesis was generally accepted that the mandibular angle did increase to some extent with tooth loss in most groups apart from SAA. The null hypothesis that the size of the mandibular angle increases with aging in all groups was rejected. In females of dentition group 2 a moderate negative correlation in the mandibular angle size existed with aging.

The widening of the mandibular angle in edentate subjects may be explained either by the absence of the molar buttress or by disequilibrium between the elevator and depressor muscles of the mandible which favors the depressors (Yanıkoğlu and Y1lmaz, 2008). The depressor muscles of the mandible include the digastrics which tend to pull the mandible towards the hyoid bone, which in turn is braced by the infrahyoid muscles. The influence of the platysma, the limiting mass of the tongue within the mouth and the effect of gravity further increase the space between the upper and lower jaws. The powerful elevating muscles of the mandible are capable of overriding the forces opposing them so as to lift the mandible in occlusal contact (Potgieter et al., 1983). In the absence of teeth, the action of the masseter and medial pterygoid muscles at the angle is not buttressed by the contact of the whole biting surface of the teeth of the mandible with that of the maxilla, but only anteriorly by the alveolar processes. The muscles of mastication will thereafter tend to mold the mandible in such a way so as to restore the parallelism of the biting surfaces, which involves an increase in the angle of the mandible (Keen, 1945). The condyle rotates posteriorly in the mandibular fossa and the rest of the mandible anteriorly to maintain contact with the maxilla anteriorly (Tallgren, 1972).

The mandibular angle of mandibles belonging to African males was the least affected by tooth loss. The greater dimensions seen in males and individuals of African ancestry could have provided some protective effect (De Villiers, 1968; Parr, 2005; Franklin et al., 2006; Franklin et al., 2008a; Franklin et al., 2008b). Apart from greater bony dimensions and associated stronger muscles of mastication a shorter duration of tooth loss before death could have been implicated as well (Patriquin, 2013). A longer possible duration of tooth loss before death could provide a longer time for the altered biomechanical forces to re-shape the mandible. Greater levels of variance (less precise estimates) were further evident from the larger confidence intervals in females as compared to males and SAE compared to SAA (Table 3). Confidence intervals of dentition groups overlapped each other within each sex- 
ancestral group. Greater levels of variance could also have been the reason why an increase in angle size between dentition groups was not significant in most sex-ancestral groups.

The tendency for the difference in mandibular angle to be accentuated between dentition groups 2 and 1, and not so much between dentition groups 1 and 0 , could be indicative that the dentition pattern of group 1 was indeed inefficient. This would fit with the proposal that unbalanced tooth loss between sides, without adequate occlusions anterior and posterior, create an inefficient mastication process (Kasai et al., 1994; Kasai et al., 1996; Kohakura et al., 1997; Ceylan et al., 1998; Pileicikiene and Surna, 2004; Yanıkoğlu and Y1lmaz, 2008; Oettlé et al., 2009).

The positive effects of wearing dentures could have confounded these relationships to some degree but unfortunately information on dentures was not available. It was thought that rehabilitation of the masticatory system after tooth extraction by denture wearing prevented the widening of the mandibular angle (Keen, 1945). In the study performed by Yanıkoğlu and Yilmaz (2008), it was found that the mandibular angle returned to the dentulous state in individuals who wore complete dentures.

When teeth were retained in pivotal positions for ongoing effective mastication, as defined before, the mandible was remodeled and strengthened to accommodate the strains of the bite force exerted on it over the years. The aging process promoted the angulation of the mandible, i.e. aging was associated with a more acute angle. This was especially true in females. This is in agreement with previous research regarding age changes in the vertical dimensions of the face from 22 to 33 years. Age changes in the vertical dimensions of the face were shown to be greater in females than in males (Bondevik, 1995; Akgül and Toygar, 2002). This could possibly be because of constructive changes in the mandible extending beyond puberty in females. In males, on the other hand, under the influence of hormones the changes in the mandible are more pronounced at puberty and might therefore not be as noticeable in our study as it only includes individuals 18 years and older (Walker and Kowalski, 1972).

\section{Conclusion}

In this study the size of the mandibular angle was assessed as a reflection of the biomechanical forces acting on the mandible on three groups showing progressive tooth loss. Apart from the effect of loss of teeth on the mandibular angle, the effect of aging was considered as well. Aging per se was found not to be associated with an increase in mandibular angle size. A decrease in angle size was noted in females with aging when 
enough teeth were retained in occlusion that had an even arrangement (two or more posterior teeth and one or more front teeth in occlusion on each side). This finding may be indicative of a constructive change beyond puberty in females. In general, an increase in size of the mandibular angle was noted with loss of teeth in all groups. However, it seemed that being male and of African ancestry provided some protective effect against the remodeling associated with tooth loss. In some groups the increase in the mandibular angle was accentuated when minimal tooth loss was associated with the disturbance of the even occlusion pattern rather than with more extensive tooth loss. This finding could support the notion that fewer than two posterior teeth and/or no front teeth in occlusion on each side were indeed inefficient. The wearing of dentures could have confounded the results in favor of those without occlusions. A limitation of this study is that dental histories were not known. Further studies could be designed to take dental records into account. From these findings it is clear that the mandibular angle that implies masticatory function varies in its response to tooth loss and aging amongst the sexes and ancestral groups. There seems to be much gained in masticatory function by retaining enough teeth in occlusion with an even arrangement, especially in females.

\section{Acknowledgements}

The authors would like to acknowledge the use of the Pretoria Bone Collection housed in the Department of Anatomy, University of Pretoria. The authors declare that they have no conflict of interest.

\section{Author Contributions}

ACO designed the study, collected the data and wrote the manuscript; RE analyzed the data and MS edited the manuscript for intellectual content and provided critical comments on the manuscript. 


\section{Literature cited}

Akgül, AA and Toygar, TU 2002. Natural craniofacial changes in the third decade of life: a longitudinal study. Am J Orthod Dentofacial Orthop 122:512-522.

Bondevik O. 1995. Growth changes in the cranial base and the face: a longitudinal cephalometric study of linear and angular changes in adult Norwegians. Eur J Orthod 17:525-532.

Ceylan G, Yaníkoglu N, Yílmaz AB and Ceylan Y. 1998. Changes in the mandibular angle in the dentulous and edentulous states. J Prosthet Dent 80:680-684.

Da Costa De Sousa J, Machado F, Silva P, Cardinot T and Babinski M. 2013. Correlation of the gonial angle with condylar measurements on dry mandible: a morphometric study for clinicalsurgical and physiotherapeutic practices. Eur J Anat 10:91-96.

De Villiers H. 1968. The skull of the South African Negro: A Biometrical and Morphological Study, Witwatersrand University Press. $342 \mathrm{p}$.

Doual J, Ferri J and Laude M. 1997. The influence of senescence on craniofacial and cervical morphology in humans. Surg Radiol Anat 19:175-183.

Enlow DH, Bianco HJ and Eklund S. 1976. The remodeling of the edentulous mandible. J Prosthet Dent 36: 685-693.

Franklin D, O'Higgins P, Oxnard CE and Dadour I. 2006. Determination of sex in South African blacks by discriminant function analysis of mandibular linear dimensions. Forensic Sci Med Pathol 2:263-268.

Franklin D, O'Higgins P, Oxnard CE and Dadour I. 2008a. Discriminant function sexing of the mandible of indigenous South Africans. Forensic Sci Int 179:84. e81-84. e85.

Franklin D, O'Higgins P and Oxnard C. 2008b. Sexual dimorphism in the mandible of indigenous South Africans: a geometric morphometric approach. S Afr J Sci 104:101-106.

Israel H. 1973. The failure of aging or loss of teeth to drastically alter mandibular angle morphology. J Dent Res 52: 83-90.

Jensen E and Palling M. 1954. The gonial angle: A survey. Am J Orthod 40:120-133.

Kasai K, Enomoto Y, Ogawa T, Kawasaki Y, Kanazawa E and Iwasawa T. 1996. Morphological Characteristics of Vertical Sections of the Mandible Obtained by CT Scanning. Anthropol. Sci, 104:187-198.

Kasai K, Richards L, Kanazawa E, Ozaki T and Iwasawa T. 1994. Relationship between attachment of the superficial masseter muscle and craniofacial morphology in dentate and edentulous humans. J Dent Res 73:1142-1149.

Keen J. 1945. A study of the angle of the mandible. J Dent Res 24:77-86.

Kohakura S, Kasai K, Ohno I and Kanazawa E. 1997. Relationship between maxillofacial morphology and morphological characteristics of vertical sections of the mandible obtained by CT scanning. J Dent Res 39:71-77.

L'Abbé E, Loots $M$ and Meiring J. 2005. The Pretoria bone collection: a modern South African skeletal sample. HOMO 56:197-205.

Moipolai P, Karic V and Miller V. 2003. The effect of the gonial angle, ramus length, age and gender on the temporomandibular opening index. J Oral Rehabil 30:1195-1199.

Moore-Jansen PH, Jantz RL and Ousley SD. 1994. Data collection procedures for forensic skeletal material, Forensic Anthropology Center, Department of Anthropology, University of Tennessee.

Morant G, Collett M and Adyanthaya N. 1936. A biometric study of the human mandible. Biometrika 84-122.

Oettlé AC, Becker PJ, De Villiers E and Steyn M. 2009. The influence of age, sex, population group, and dentition on the mandibular angle as measured on a South African sample. Am J Phys Anthropol 139:505-511.

Ohm E and Silness J. 1999. Size of the mandibular jaw angle related to age, tooth retention and gender. J Oral Rehabil 26:883-891. 
Osato S, Kuroyama I, Nakajima S, Ogawa T and Misaki K. 2012. Differences in 5 anatomic parameters of mandibular body morphology by gonial angle size in dentulous Japanese subjects. Ann Anat 194:446-451.

Parr NM. 2005. Determination of Ancestry from Discrete Traits of the Mandible. University of Indianapolis. $106 \mathrm{p}$.

Patriquin ML. 2013. The relationship between masticatory stress and prognathism: a finite element and morphometric study. $277 \mathrm{p}$.

Pileicikiene $G$ and Surna A. 2004. The human masticatory system from a biomechanical perspective: A review. Stomatologija 6:81-84.

Potgieter PJ, Monteith BD and Kemp PL. 1983. The determination of free-way space in edentulous patients: a cephalometric approach. J Oral Rehabil 10:283-293.

Srisopark S. 1975. Postnatal growth of the mandible as revealed by a measuring technique on the mandibulometer. J Dent Assoc Thai 25:19-30.

Tallgren A. 1972. The continuing reduction of the residual alveolar ridges in complete denture wearers: a mixed-longitudinal study covering 25 years. J Prosthet Dent, 27:120-132.

Tobias PV. 1974. The biology of the southern African Negro. In: Hammond-Tooke WD, editor. The Bantu-speaking peoples of Southern Africa. London and Boston: Routledge and Kegan Paul, p 3-45.

Walker GF and Kowalski CJ. 1972. On the growth of the mandible. Am J Phys Anthropol 36:111-117.

Xie Q-F and Ainamo A. 2004. Correlation of gonial angle size with cortical thickness, height of the mandibular residual body, and duration of edentulism. J Prosthet Dent 91:477-482.

Yanıkoğlu N and Yılmaz B. 2008. Radiological evaluation of changes in the gonial angle after teeth extraction and wearing of dentures: a 3-year longitudinal study. Oral Surg Oral Med Oral Pathol Oral Radiol Endod 105:e55-e60. 